\title{
Direct Reactions with Exotic Nuclei, Nuclear Structure and Astrophysics
}

\author{
G.Baur, ${ }^{1}$ S.Typel ${ }^{2}$ \\ ${ }^{1}$ Institut für Kernphysik, Forschungszentrum Jülich \\ D-52425 Jülich, Germany \\ ${ }^{2}$ Gesellschaft für Schwerionenforschung mbH(GSI), Planckstrasse 1 \\ D-64291 Darmstadt, Germany
}

February 9, 2008

\begin{abstract}
Intermediate energy Coulomb excitation and dissociation is a useful tool for nuclear structure and astrophysics studies. Low-lying strength in nuclei far from stability was discovered by this method. The effective range theory for low-lying strength in one-neutron halo nuclei is summarized and extended to two-neutron halo nuclei. This is of special interest in view of recent rather accurate experimental results on the low-lying electric dipole strength in ${ }^{11} \mathrm{Li}$. Another indirect approach to nuclear astrophysics is the Trojan horse method. It is pointed out that it is a suitable tool to investigate subthreshold resonances.
\end{abstract}

\section{Introduction and Overview}

With the exotic beam facilities all over the world - and more are to come - direct reaction theories are experiencing a renaissance. These direct reaction theories are essential to interprete indirect methods used in nuclear astrophysics. We discuss Coulomb dissociation 1 and the Trojan horse method, for a recent review see [2]. Coulomb dissociation was reviewed in 2003 [3], a comprehensive paper on the Trojan horse method appeared in [4. A more recent (mini)review of indirect methods can be found in 5]. Here we report on the effective range theory of low-lying electromagnetic strength in one-nucleon halo nuclei [6, 7, and its extension to two-neutron halo nuclei. This will be of special interest in view of recent rather accurate experimental results on the low-lying electric dipole strength in ${ }^{11} \mathrm{Li}[8]$.

The Trojan-Horse Method [4 is a particular case of transfer reactions to the continuum under quasi-free scattering conditions. Special attention is paid to the transition from reactions to bound and unbound states and the role of subthreshold resonances 9]. Since the binding energies of nuclei close to the drip line tend to be small, this is expected to be an important general feature in exotic nuclei.

\section{Effective Range Theory of Halo Nuclei}

\subsection{Single particle halo nuclei}

At low energies the effect of the nuclear potential is conveniently described by the effective-range expansion [10. An effective-range approach for the electromagnetic strength distribution in neutron 
halo nuclei was introduced in [6, 7] and applied to the single neutron halo nucleus ${ }^{11}$ Be. Recently, the same method was applied to the description of electromagnetic dipole strength in ${ }^{23} \mathrm{O}[11$. A systematic study sheds light on the sensitivity of the electromagnetic strength distribution to the interaction in the continuum. We expose the dependence on the binding energy of the nucleon and on the angular momentum quantum numbers. Our approach extends the familiar textbook case of the deuteron, that can be considered as the prime example of a halo nucleus, to arbitrary nucleon+core systems, see also [12. We also investigate in detail the square-well potential model. It has great merits: it can be solved analytically, it shows the main characteristic features and it leads to rather simple and transparent formulae. As far as we know, some of these formulae have not been published before. These explicit results can be compared to our general theory for low energies (effective-range approach) and also to more realistic Woods-Saxon models. Due to shape independence, the results of these various approaches will not differ for low energies. It will be interesting to delineate the range of validity of the simple models.

Our effective-range approach is closely related to effective field theories that are nowadays used for the description of the nucleon-nucleon system and halo nuclei [13. The characteristic low-energy parameters are linked to QCD in systematic expansions. Similar methods are also used in the study of exotic atoms $\left(\pi^{-} A, \pi^{+} \pi^{-}, \pi^{-} p, \ldots\right)$ in terms of effective-range parameters. The close relation of effective field theory to the effective-range approach for hadronic atoms was discussed in Ref. [14].

In the ANC method [15] it is assumed that the radial continuum wave function $g_{l_{f}}$ is a pure Coulomb wave function (a regular spherical Bessel function for neutrons) and that the dominant contribution to the radial integral

$$
I_{r}=\int_{0}^{\infty} f_{l_{i}}(r) r g_{l_{f}}(r) d r
$$

comes from the region $r>R$ where $\mathrm{R}$ is the range of the nuclear interaction. In this case the $\mathrm{S}$-factor is entirely determined by the asymptotic normalization constant (ANC) $C_{l_{i}}$ of the bound state wave function

$$
f_{l_{i}}(r) \sim C_{l_{i}} e^{-q r}
$$

where $l_{i}$ is the orbital angular momentum. The ANC is determined by a suitable transfer reaction. In some cases this is a good approximation. In general there is an influence from the final state interaction on the final continuum wave function. In the outside region $(r>R)$ it is given by

$$
g_{l_{f}}=F_{l_{f}} \cos \delta_{l_{f}}+G_{l_{f}} \sin \delta_{l_{f}}
$$

where the regular and irregular Coulomb wave functions are denoted by $F_{l_{f}}$ and $G_{l_{f}}$ respectively. For the low energies relevant now, the phase shift $\delta_{l_{f}}$ is given by the low energy parameters of the effective range expansion. Usually this is one number, the scattering length.

In Fig. 11 we show the application of the method to the electromagnetic dipole strength in ${ }^{11} \mathrm{Be}$. The reduced transition probability was deduced from high-energy ${ }^{11}$ Be Coulomb dissociation at GSI [16]. Using a cutoff radius of $R=2.78 \mathrm{fm}$ and an inverse bound-state decay length of $q=0.1486 \mathrm{fm}^{-1}$ as input parameters we extract an $\mathrm{ANC}$ of $C_{0}=0.724(8) \mathrm{fm}^{-1 / 2}$ from the fit to the experimental data. The ANC can be converted to a spectroscopic factor of $C^{2} S=0.704(15)$ that is consistent with results from other methods. In the lowest order of the effective-range expansion the phase shift in the partial wave with orbital angular momentum $l$ and total angular momentum $j$ is written as $\tan \delta_{l}^{j}=-\left(x c_{l}^{j} \gamma\right)^{2 l+1}$. The halo expansion parameter is denoted by $\gamma=q R=0.4132<1$ and $x=k / q=\sqrt{E / S_{n}}$ where the neutron separation energy is given by $S_{n}$. The effective range term $\frac{1}{2} r_{l} k^{2}$ term can be neglected, since it leads to a contribution with an extra $\gamma^{2}$ factor which is small in the halo nucleus limit $\gamma \rightarrow 0$ (at least in the case where the scattering length and the effective range parameter are of natural order). The dimensionless parameter $c_{l}^{j}$ corresponds to the scattering length $a_{l}^{j}=\left(c_{l}^{j} R\right)^{2 l+1}$. We obtain $c_{1}^{3 / 2}=-0.41(86,-20)$ and $c_{1}^{1 / 2}=2.77(13,-14)$, corresponding to $a_{1}^{1 / 2}=456(61,-73) \mathrm{fm}^{3}$. This unnaturally large value was 


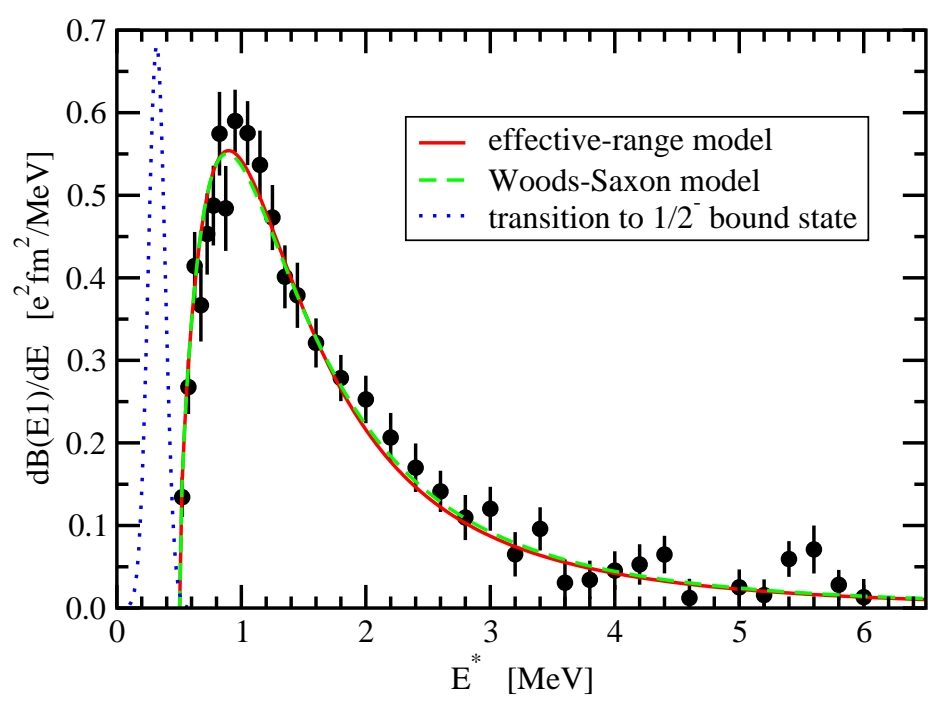

Figure 1: The reduced electric dipole transition probability is shown as a function of the excitation energy $E^{*}=E+S_{n}$. The theoretically calculated dipole strength is compared to experimental data extracted from the Coulomb dissociation of ${ }^{11} \mathrm{Be}$ [16].

found directly from the analysis of Coulomb dissociation data [16. It finds a natural explanation in the existence of the $\frac{1}{2}^{-}$-bound state close to the neutron breakup threshold in ${ }^{11} \mathrm{Be}$.

The connection of the scattering length $a_{l}$ and the bound state parameter $\mathrm{q}$ for $l>0$ is given by $a_{l}=\frac{2(2 l-1) R^{2 l-1}}{q^{2}(2 l+1) !(2 l-1) ! !}$ in a square well model with radius $\mathrm{R}$. This is a generalization of the well-known relation $a_{0}=1 / q$ for $l=0$. The $p_{1 / 2}$ channel in ${ }^{11} \mathrm{Be}$ is an example for the influence of a halo state on the continuum. The binding energy of this state is given by $184 \mathrm{keV}$, which corresponds to $q=0.094 \mathrm{fm}^{-1}$. With $R=2.78 \mathrm{fm}$ one has $\gamma^{2}=0.068$. For $l=1$ one has $a_{1}=\frac{2 R^{3}}{3 \gamma^{2}}=210 \mathrm{fm}^{3}$. Applications to low-lying single neutron halo strength in carbon isotopes are given in [17.

Let us now mention very briefly the proton case 7]. Only a numerical approach is possible now. Again one finds that the ANC is not the only factor which determines the S-factor: there is also an influence of the scattering length. In Fig. 2 the influence of the potential depth $V_{0}$ on the strength distribution is shown. We refer to [7] for further discussion.

\subsection{Two neutron halo nuclei, application to ${ }^{11} \mathrm{Li}$}

While single particle halos show rather simple features which can largely be explained in the frame of the one-body Schrödinger equation, two neutron (or two proton, or neutron-proton) halo nuclei show many more interesting aspects.

A Borromean nucleus is a three-body system which falls apart when one of the particles is removed, ${ }^{11} \mathrm{Li}$ is an example. It can be considered as a bound system of two neutrons and a core $\left({ }^{9} \mathrm{Li}\right)$. The system is barely bound $\left(E_{b} \sim 300 \mathrm{keV}\right)$ and the core can be assumed to be inert. The subsystems (n-n) and $\left({ }^{9} \mathrm{Li}-\mathrm{n}\right)$ are unbound. The binding of the Borromean nucleus is due to the attractive n-n interaction which leads to the binding of the three-body system, see e.g. [18].

Essentially due to the small binding energy the low-lying E1 strength shows simple universal features as we will show now. The system is probed by long wavelength photons and can be characterized by a few low energy constants. As we have seen in the previous subsection, the dipole matrixelement is dominated by contributions from distances larger than the range of the core-neutron interaction. In this region the wave function depends on the binding energy $E_{b}$. In a mean field approach the binding 


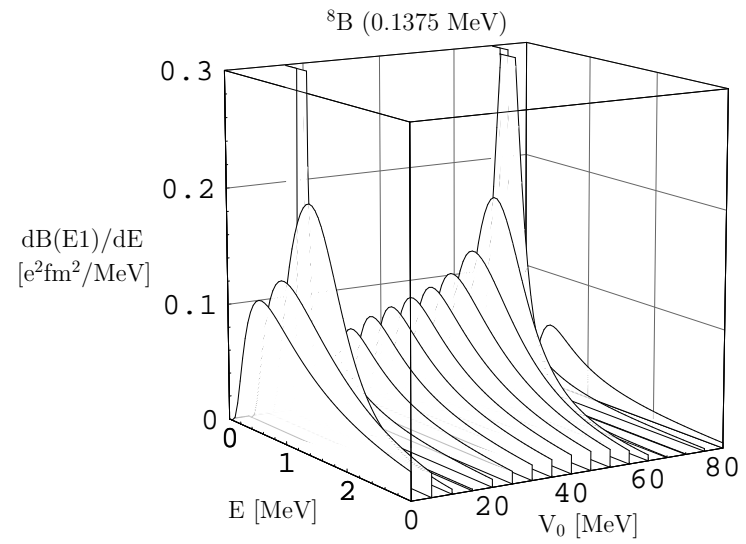

Figure 2: Reduced transition probablity $d B(E 1) / d E$ for the breakup of ${ }^{8} \mathrm{~B}$ into a proton and ${ }^{7} \mathrm{Be}$ as a function of the relative energy $\mathrm{E}$ of the final state for various depths $V_{0}$ of the potential in the continuum. The proton separation energy in ${ }^{8} \mathrm{~B}$ is given by $0.1375 \mathrm{MeV}$. For further explanations see [7].

energy is shared equally between the two neutrons. Each neutron is bound by $\epsilon=\frac{\hbar^{2} q^{2}}{2 m}=E_{b} / 2$. The slope of the wave function is characterized by $q$, the overall magnitude by an asymptotic normalization constant $C_{l}: \phi_{l, \epsilon}(r) \rightarrow C_{l} e^{-q r}$.

For simplicity let us neglect spin-orbit forces and assume that the neutron spin state is the antisymmetric $S=0$ state. The space part is symmetric, we include $s^{2}$ and $p^{2}$ configurations, with the corresponding spectroscopic factors $C^{2} S_{l}$. The radial part of the two-neutron halo wave function is written as $\sim b_{l} \phi_{l, \epsilon}\left(r_{1}\right) \phi_{l, \epsilon}\left(r_{2}\right)$. The amplitudes $b_{l}$ are related to the spectroscopic factors by $C^{2} S_{l}=\left|b_{l}\right|^{2}$. The wave function for $r_{1,2}>R$ is rather simple, it must be borne in mind that the binding itself is caused by the full three-body dynamics.

The dipole operator is a one-body operator. It is given in terms of the Jacobi coordinates $\vec{\rho}_{1}$ and $\vec{\rho}_{2}$ by

$$
M(E 1 \mu)=Z_{\mathrm{eff}} e\left(\rho_{1} Y_{1 \mu}\left(\hat{\rho}_{1}\right)+\rho_{2} Y_{1 \mu}\left(\hat{\rho}_{2}\right)\right)
$$

The introduction of the Jacobi coordinates introduces an asymmetry between the two identical neutrons. This translates into an asymmetry of the effective charges for both neutrons, we neglect this effect, which is of the order of $1 / A_{c}$, where $A_{c}$ is the mass of the core. For the effective charge we take $Z_{\text {eff }}=\frac{-Z_{c} m_{n}}{m_{n}+m_{c}}$. (The other possible choice would be $Z_{\text {eff }}=\frac{-Z_{c} m_{n}}{2 m_{n}+m_{c}}$.) The final state is given by two continuum neutrons with momenta $\vec{k}_{1}$ and $\vec{k}_{2}$. We assume that they are uncorrelated. Low lying strength is a long wavelength phenomenon, and the interaction can be characterized by the n-core scattering lengths $a_{0}$ and $a_{1}$ in the $l=0$ and $l=1$ channels. We know that ${ }^{10} \mathrm{Li}$ is unbound, so these scattering lengths are negative. The continuum radial wave functions are given by a product of single particle wave functions of the type given by eq. 3 above.

The dipole matrix element consists of a product of two terms: one is characterized by the dipole selection rule $\Delta l=1$ and is of the type encountered in the single particle case above. We have

$$
\frac{d B(E 1)}{d E}=Z_{e f f}^{2} \frac{2 m}{\pi \hbar^{2}} \frac{3}{4 \pi}\left(l_{i} 010 \mid l_{f} 0\right)^{2} \frac{C_{l}^{2}}{q^{5}} S_{l_{i}}^{l_{f}}
$$

where the shape functions [6] are given in terms of the dimensionless quantity $x=\frac{k}{q}$ by

$$
S_{0}^{1}(\lambda=1)=\frac{4 x^{3}}{\left(1+x^{2}\right)^{4}}\left(1-a_{1} q^{3}\left(1+3 x^{2}\right)+\ldots\right)
$$


for s to p-transitions and by

$$
S_{1}^{0}(\lambda=1)=\frac{x\left(3+x^{2}\right)^{2}}{\left(1+x^{2}\right)^{4}}\left(1-\frac{4 a_{0} q}{3+x^{2}}+\ldots\right)
$$

for p-to s-transitions.

The other term is an overlap term

$$
\frac{d N}{d E}=\frac{2}{\pi} \frac{m}{\hbar^{2} k}\left|<\phi_{l \epsilon}\right| \phi_{l \vec{k}}>\left.\right|^{2}
$$

where $E=\frac{\hbar^{2} k^{2}}{2 m}$ (using the normalization conventions of [7]). One can describe the physics of this matrixelement in quite simple terms: Due to the sudden removal of one of the neutrons by the interaction of the photon with the core, the remaining neutron finds itself in the field of the ${ }^{9} \mathrm{Li}$ core, where it is no longer bound. A 'shake off' occurs, which is described by the overlap term, eq.9. It is non-zero, because the two wave functions belong to different Hamiltonians. A similar and familiar example is the shake-off of electrons after $\beta$-decay, where the nuclear charge is suddenly changed by one unit. This approach can also be applied to non-Borromean nuclei. In this case the neutron can also be left in a bound state with a certain probability which can be obtained from the corresponding overlap matrixelement.

The overlap integral is strongly peaked around zero energy. For $l=0$ one obtains

$$
\left|<\Phi_{0 \epsilon}\right| \Phi_{0 k}>\left.\right|^{2}=\frac{2 q k^{2}\left(a_{0} q-1\right)^{2}}{\left(1+\left(a_{0} k\right)^{2}\right)\left(q^{2}+k^{2}\right)^{2}}
$$

In the unitary limit $\left(a_{0} \rightarrow \infty\right)$ this reduces to

$$
\left|<\Phi_{0 \epsilon}\right| \Phi_{0 k}>\left.\right|^{2}=\frac{2 q k^{2}}{\left(q^{2}+k^{2}\right)^{2}}
$$

This is probably a good approximation for the overlap term in the ${ }^{11} \mathrm{Li}$ case where one neutron is almost bound to the ${ }^{9} \mathrm{Li}$ core. The analytical formula for all values of the angular momentum is given in Appendix A.4 of [7] $(\lambda=0)$.

Low-lying E1 strength in ${ }^{11} \mathrm{Li}$ was observed experimentally in Ref. [8]. The dipole strength distribution as a function of the relative energy $E=E_{1}+E_{2}=\frac{\hbar^{2}}{2 m}\left(k_{1}^{2}+k_{2}^{2}\right)$ of the ${ }^{9}$ Li-n-n-system is shown in Fig.3 there. It will be interesting to compare this experimental result to the present approach. The dipole strength distribution $\frac{d B(E 1)_{2 n} \text {-halo }}{d E}$ depending on the relative energy E can be obtained by folding the dipole distribution with the overlap term:

$$
\frac{d B(E 1)_{2 \mathrm{n}-\text { halo }}}{d E}=2 \iint d E_{1} d E_{2} \frac{d B E 1}{d E_{2}} \frac{d N}{d E_{1}} \delta\left(E-\left(E_{1}+E_{2}\right)\right)
$$

The factor of 2 arises because there are two neutrons in the same shell. We assume that the binding energy $E_{b}$ is known. The shapes of the functions $S_{0}^{1}$ and $S_{1}^{0}$ (see eqs. 7 and 8) (and $S_{1}^{2}$ ) show characteristic differences, so the fit to the experimental results [8] will be quite sensitive to the spectroscopic factors $C^{2} S_{0}$ and $C^{2} S_{1}$. The width of the nonorthogonality term depends on the scattering lengths, so they can also be determined by this 4-parameter fit, but probably one will be less sensitive to these quantities. The experimental results [8] are quite well explained by the theory of Esbensen and Bertsch [19]. It will be interesting to compare the present approach also to the more detailed theory of [19]. In the present approach only a few low energy parameters like the scattering lengths in the relevant channels enter. 


\section{Trojan Horse Method}

A similarity between cross sections for two-body and closely related three-body reactions under certain kinematical conditions [20] led to the introduction of the Trojan-Horse method [21, 22, 23, 4]. In this indirect approach a two-body reaction

$$
A+x \rightarrow C+c
$$

that is relevant to nuclear astrophysics is replaced by a reaction

$$
A+a \rightarrow C+c+b
$$

with three particles in the final state. One assumes that the Trojan horse $a$ is composed predominantly of clusters $x$ and $b$, i.e. $a=(x+b)$. This reaction can be considered as a special case of a transfer reaction to the continuum. It is studied experimentally under quasi-free scattering conditions, i.e. when the momentum transfer to the spectator $b$ is small. The method was primarily applied to the extraction of the low-energy cross section of reaction (12) that is relevant for astrophysics. However, the method can also be applied to the study of single-particle states in exotic nuclei around the particle threshold.

\subsection{Continuous Transition from positive to negative energies in the $(\mathrm{A}+\mathrm{x})-$ channel}

Motivated by this we look again at the relation between transfer to bound and unbound states. We study the reaction $A+a \rightarrow B+b$ where $a=(b+x)$ and $\mathrm{B}$ denotes the final $B=(A+x)$ system. It can be a bound state $B$ with binding energy $E_{\text {bind }}=-E_{A x}(>0)$, the open channel $A+x$, with $E_{A x}>0$, or another channel $C+c$ of the system $B=(A+x)$. In particular, the reaction $x+A \rightarrow C+c$ can have a positive $Q$ value and the energy $E_{A x}$ can be negative as well as positive. As an example we quote the recently studied Trojan horse reaction $d+{ }^{6} \mathrm{Li} 24$ applied to the ${ }^{6} \mathrm{Li}(p, \alpha){ }^{3} \mathrm{He}$ two-body reaction (the neutron being the spectator). In this case there are two charged particles in the initial state $\left({ }^{6} \mathrm{Li}+p\right)$. Another example with a neutral particle $x$ would be ${ }^{10} \mathrm{Be}+d \rightarrow p+{ }^{11} \mathrm{Be}+\gamma$. The general question which we want to answer here is how the two regions $E_{A x}>0$ and $E_{A x}<0$ are related to each other. E.g., in Fig. 7 of 24] the coincidence yield is plotted as a function of the ${ }^{6} \mathrm{Li}-p$ relative energy. It is nonzero at zero relative energy. How does the theory 4] (and the experiment) continue to negative relative energies? With this method, subthreshold resonances can be investigated rather directly.

The Trojan Horse amplitude $\mathrm{f}$ involves the combination $f \propto S_{l c} \cdot J_{l}^{+}$(see eq. (61) of [4]). In this reference, only the case $E \equiv E_{A x}>0$ is dealt with explicitly. The threshold behaviour of $J_{l}^{+}$in the case of neutrons is given by (A.30), for charged particles by eq. (59) of [4, respectively. $S_{l c}$ is the S-matrixelement connecting the channel $c \equiv c+C$ and the elastic channel $A+x$ with angular momentum $l$. For neutral particles the threshold behaviour is $\left|S_{l c}\right|^{2} \sim(k R)^{2 l+1}$ and the cross section $\sigma \propto k^{-3}\left|S_{l c} J_{l}^{+}\right|^{2}$ tends to a finite constant. A similar analysis can be done for charged particles. Let us now study the case $E_{A x}<0$ and the transition from $E_{A x}>0$ to $E_{A x}<0$. In the latter case the wave number $\mathrm{k}$ becomes complex, $\mathrm{k}=i \mathrm{q}$ and the factor $J_{l}^{+}$involves a decaying function, instead of the oscillatory one. The quantity $S_{l c}$ is no longer an S-matrixelement, it is the normalization factor for the decaying wave-function. The continuous transition for both cases was studied in 9 in a two-channel model.

An interesting case is when there are subthreshold resonances, like in the systems ${ }^{14} N(p, \gamma){ }^{15} \mathrm{O}$ and in ${ }^{12} C(\alpha, \gamma){ }^{16} O$. In [25, 9] a two-channel model is studied.The standard Breit-Wigner result is obtained

$$
S_{i j}=e^{i\left(\xi_{i}+\xi_{f}\right)}\left(\delta_{i j}-\frac{i \sqrt{\Gamma_{i} \Gamma_{j}}}{E-E_{R}+i \Gamma / 2}\right)
$$

where $E_{R}$ denotes the resonance energy, the partial widths are given by $\Gamma_{1}$ and $\Gamma_{2}$, the total width is given by $\Gamma=\Gamma_{1}+\Gamma_{2}$. 
For $E<0$ the width $\Gamma_{1}$ turns out to be imaginary [9]. For $E_{R}>0$ we have a resonance, for $E_{R}<0$ a subthreshold resonance, the formulae are valid for both cases. In [26, 27] sub-Coulomb $\alpha$ transfer is used to study the low energy ${ }^{12} C(\alpha, \gamma){ }^{16} O$ S-factor. Transitions to the $1^{-}$and $2^{+}$bound states are observed and the reduced $\alpha$ widths of these states is obtained.

\section{Conclusion}

While the foundations of direct reaction theory have been laid several decades ago, the new possibilites which have opened up with the rare isotope beams are an invitation to revisit this field. The general frame is set by nonrelativistic many-body quantum scattering theory, however, the increasing level of precision demands a good understanding of relativistic effects notably in intermediate-energy Coulomb excitation.

The properties of halo nuclei depend very sensitively on the binding energy and despite the ever increasing precision of microscopic approaches using realistic NN forces it will not be possible, say, to predict the binding energies of nuclei to a level of about $100 \mathrm{keV}$. Thus halo nuclei ask for new

approaches in terms of some effective low-energy constants. Such a treatment was provided in Ch. 2 and an example with the one-neutron halo nucleus ${ }^{11} \mathrm{Be}$ was given. With the future radioactive beam facilities at RIKEN and GSI one will be able to study also neutron halo nuclei for intermediate masses in the years to come. This is expected to be relevant also for the astrophysical r-process. It is a great challenge to extend the present approach for one-nucleon halo nuclei to more complicated cases, like two-neutron halo nuclei. In the present work we obtained a rather simple formula for two-neutron halo nuclei with a transparent interpretation: after the first neutron is removed, the second one is left in a different field (Hamiltonian) which leads to a shake-off process, similar to the rearrangement of the atomic cloud after an $\alpha$ - or $\beta$ decay.

While the Coulomb dissociation method relies essentially only on QED, precise experiments can give, in combination with a thorough theoretical analysis, precise answers for the astrophysical S factors. In the Trojan-Horse Method more phenomenological aspects enter, like optical model parameters and effective nuclear interactions. This makes the interpretation of the experimental results in terms of astrophysical $\mathrm{S}$ factors less precise. In the Trojan-Horse Method one can extract bare nucleus (unscreened) S-factors, see e.g. 24]. In the interpretation of screening effects one relies on the accuracy of the energy dependence. This is certainly better fulfilled than the accuracy of the absolute values.

\section{References}

[1] G. Baur, C. A. Bertulani, and H. Rebel, Nucl. Phys., A548, 188 (1986).

[2] S. Typel Eur. Phys. J. A 25, 665 (2005)

[3] G. Baur, K. Hencken, and D. Trautmann, Progress in Particle and Nuclear Physics, 51, 487 (2003).

[4] S. Typel and G. Baur, Ann. Phys., 305, 228 (2003).

[5] G. Baur and S. Typel nucl-th/0601004, Proceedings of the DAE-BRNS 50th Symposium on Nuclear Physics, Bhabha Atomic Research Centre, Mumbai, India December 12-16, 2005

[6] S. Typel and G. Baur, Phys. Rev. Lett., 93, 142502 (2004).

[7] S. Typel and G. Baur, Nucl. Phys. A759, 247 (2005), nucl-th/0411069.

[8] T. Nakamura et al. Phys. Rev. Lett. 96, (2006)25202 
[9] G. Baur and S. Typel, Proceedings of Fusion06, San Servolo, Italy, International Conference on Reaction Mechanisms and Nuclear Structure at the Coulomb Barrier, S.Servolo Island (Venezia)Italy March 19-23, 2006 CP853, FUSION06, edited by L.Corradi et al., 2006 American Institute of Physics pgs. 366-371, nucl-th/0604057

[10] H. A. Bethe, Phys. Rev., 76, 38 (1949).

[11] C. Nociforo et al., Phys. Lett. B, 605, 75 (2005).

[12] D. M. Kalassa and G. Baur, J. Phys. G, 22, 115 (1996).

[13] C. A. Bertulani, H.-W. Hammer, and U. van Kolck, Nucl. Phys. A, 721, 37 (2002).

[14] B. R. Holstein, Phys. Rev. D, 60, 114030 (1999).

[15] A. M. Mukhamedzhanov, R. E. Tribble, N. K. Timofeyuk Phys. Rev. C, 51, 3472 (1995)

[16] R. Palit et al., Phys. Rev. C, 68, 034318 (2003)

[17] U. Datta Pramanik et al. Phys. Lett. B, 551, 63 (2003), J. Phys. G, 31, S1583 (2005)

[18] A.B. Migdal Sov. J. of Nucl. Phys. 16(1973)238

[19] H. Esbensen and G. F. Bertsch Nucl. Phys., A542, 310 (1992)

[20] H. Fuchs et al., Phys. Lett. B, 37, 285 (1971).

[21] G. Baur, Phys. Lett. B, 178, 135 (1986).

[22] G. Baur, F. Rösel, D. Trautmann, and R. Shyam, Phys. Rep., 111, 333 (1984).

[23] S. Typel and H. H. Wolter, Few Body Systems, 29, 75 (2000).

[24] A. Tumino et al., Phys. Rev. C, 67, 065803 (2003).

[25] G. Baur, S. Typel Direct reactions with exotic nuclei CP791 AIP Proceedings of Reaction Mechanisms for Rare Isotope Beams, p.61 East Lansing, Michigan State University 9-12 March 2005, nucl-th/0504068

[26] C. R. Brune et al. Phys. Rev. Lett., 83, 4025 (1999).

[27] C. R. Brune et al. Nucl. Phys. A, 688, 263c (2001). 